\title{
ITERATED HARDY-TYPE INEQUALITIES INVOLVING SUPREMA
}

\section{Amiran Gogatishvili And RzA CH. Mustafayev}

Abstract. In this paper, the boundedness of the composition of the supremal operators defined, for a non-negative measurable functions $f$ on $(0, \infty)$, by

$$
S_{u} g(t):=\underset{0<\tau \leqslant t}{\operatorname{ess} \sup } u(\tau) g(\tau), t \in(0, \infty),
$$

and

$$
S_{u}^{*} g(t):=\underset{t \leqslant \tau<\infty}{\operatorname{ess} \sup } u(\tau) g(\tau), t \in(0, \infty),
$$

where $u$ is a fixed continous weight on $(0, \infty)$, with the Hardy and Copson operators between weighted Lebesgue spaces $L^{p}(v)$ and $L^{q}(w)$ are characterized.

The complete solution of the restricted inequalities, that is, inequalities

$$
\left\|S_{u}(f)\right\|_{q, w,(0, \infty)} \leqslant c\|f\|_{p, v,(0, \infty)},
$$

and

$$
\left\|S_{u}(f)\right\|_{q, w,(0, \infty)} \leqslant c\|f\|_{p, v,(0, \infty)}
$$

being satisfied on the cones of monotone functions $f$ on $(0, \infty)$, are given.

Moreover, the complete characterization of the inequality

$$
\left\|T_{u, b} f\right\|_{q, w,(0, \infty)} \leqslant c\|f\|_{p, v,(0, \infty)},
$$

being satisfied for every non-negative and non-increasing functions $f$ on $(0, \infty)$, is given for $0<p, q<\infty$, as well. Here the operator $T_{u, b}$ is defined for a measurable non-negative function $f$ on $(0, \infty)$ by

$$
\left(T_{u, b} g\right)(t):=\sup _{t \leqslant \tau<\infty} \frac{u(\tau)}{B(\tau)} \int_{0}^{\tau} g(s) b(s) d s, \quad t \in(0, \infty),
$$

where $u, b$ are two weight functions on $(0, \infty)$ such that $u$ is continuous on $(0, \infty)$ and the function $B(t):=\int_{0}^{t} b(s) d s$ satisfies $0<B(t)<\infty$ for every $t \in(0, \infty)$.

Mathematics subject classification (2010): 26D10, 26D15.

Keywords and phrases: Quasilinear operators, iterated Hardy inequalities, weights.

\section{REFERENCES}

[1] V. I. Burenkov, A. Gogatishvili, V. S. Guliyev, R. Ch. Mustafayev, Boundedness of the fractional maximal operator in local Morrey-type spaces, Complex Var. Elliptic Equ. 55 (2010), no. 8-10, 739-758, MR2674862 (2011f:42015).

[2] V. I. Burenkov, A. Gogatishvili, V. S. Guliyev, R. Ch. Mustafayev, Boundedness of the Riesz potential in local Morrey-type spaces, Potential Anal. 35 (2011), no. 1, 67-87, MR2804553 (2012d:42027).

[3] V. I. Burenkov, R. Oinarov, Necessary and sufficient conditions for boundedness of the Hardytype operator from a weighted Lebesgue space to a Morrey-type space, Math. Inequal. Appl. 16 (2013), no. 1, 1-19, MR3060376.

[4] M. Carro, L. Pick, J. Soria, V. D. StePanov, On embeddings between classical Lorentz spaces, Math. Inequal. Appl. 4 (2001), no. 3, 397-428, DOI 10.7153/mia-04-37, MR1841071 (2002d:46026). 
[5] M. J. Carro, J. Soria, Boundedness of some integral operators, Canad. J. Math. 45 (1993), no. 6, 1155-1166, DOI 10.4153/CJM-1993-064-2, MR1247539 (95d:47064).

[6] A. CiAnChi, R. KeRman, B. OPIC, L. PiCK, A sharp rearrangement inequality for the fractional maximal operator, Studia Math. 138 (2000), no. 3, 277-284, MR1758860 (2001h:42029).

[7] M. CWikel, E. Pustylnik, Weak type interpolation near “endpoint” spaces, J. Funct. Anal. 171 (2000), no. 2, 235-277, DOI 10.1006/jfan.1999.3502, MR1745635 (2001b:46118).

[8] R. YA. DoKTORSKII, Reiterative relations of the real interpolation method, Dokl. Akad. Nauk SSSR 321 (1991), no. 2, 241-245, (Russian), English transl., Soviet Math. Dokl. 44 (1992), no. 3, 665-669, MR1153547 (93b:46143).

[9] D. E. Edmunds, B. OpIC, Boundedness of fractional maximal operators between classical and weak-type Lorentz spaces, Dissertationes Math. (Rozprawy Mat.) 410 (2002), 50, DOI 10.4064/dm410-0-1, MR1952673 (2004c:42040).

[10] D. E. Edmunds, B. OpIC, Alternative characterisations of Lorentz-Karamata spaces, Czechoslovak Math. J. 58 (133) (2008), no. 2, 517-540, DOI 10.1007/s10587-008-0033-8, MR2411107 (2009c:46044).

[11] W. D. Evans, B. OPIC, Real interpolation with logarithmic functors and reiteration, Canad. J. Math. 52 (2000), no. 5, 920-960, DOI 10.4153/CJM-2000-039-2, MR1782334 (2001i:46115).

[12] A. Gogatishvili, R. Ch. Mustafayev, L.-E. Persson, Some new iterated Hardy-type inequalities, J. Funct. Spaces Appl., (2012), Art. ID 734194, 30, MR3000818.

[13] A. Gogatishvili, R. Ch. Mustafayev, L.-E. Persson, Some new iterated Hardy-type inequalities: the case $\theta=1$, J. Inequal. Appl., posted on 2013, 29 pp., DOI 10.1186/1029-242X-2013-515.

[14] A. Gogatishvili, R. CH. Mustafayev, Weighted iterated Hardy-type inequalities, Math. Inequal. Appl. (to appear).

[15] A. Gogatishvili, B. Opic, L. PICK, Weighted inequalities for Hardy-type operators involving suprema, Collect. Math. 57 (2006), no. 3, 227-255, MR2264321 (2007g:26019).

[16] A. Gogatishvili, L. Pick, A reduction theorem for supremum operators, J. Comput. Appl. Math. 208 (2007), no. 1, 270-279, DOI 10.1016/j.cam.2006.10.048, MR2347749 (2009a:26013).

[17] A. Gogatishvili, V. D. Stepanov, Reduction theorems for weighted integral inequalities on the cone of monotone functions, Uspekhi Mat. Nauk 68 (2013), no. 4 (412), 3-68, (Russian, with Russian summary), English transl., Russian Math. Surveys 68 (2013), no. 4, 597-664, MR3154814.

[18] R. Kerman, L. Pick, Optimal Sobolev imbeddings, Forum Math. 18 (2006), no. 4, 535-570, DOI 10.1515/FORUM.2006.028, MR2254384 (2007g:46052).

[19] M. Ǩ̌EPELA, Integral conditions for Hardy-type operators involving suprema, Preprint MATHKMA-2015/503: http://msekce.karlin.mff.cuni.cz/ms-preprints/kma-preprints (2015).

[20] B. OPIC, On boundedness of fractional maximal operators between classical Lorentz spaces, Function spaces, differential operators and nonlinear analysis, (Pudasjärvi, 1999), Acad. Sci. Czech Repub., Prague, 2000, 187-196, MR1755309 (2001g:42043).

[21] L. PICK, Supremum operators and optimal Sobolev inequalities, Function spaces, differential operators and nonlinear analysis, (Pudasjärvi, 1999), Acad. Sci. Czech Repub., Prague, 2000, 207-219, MR1755311 (2000m:46075).

[22] L. PICK, Optimal Sobolev embeddings - old and new, Function spaces, interpolation theory and related topics (Lund, 2000), de Gruyter, Berlin (2002), 403-411, MR1943297 (2003j:46054).

[23] D. V. Prokhorov, V. D. Stepanov, On weighted Hardy inequalities in mixed norms, Proc. Steklov Inst. Math. 283 (2013), 149-164.

[24] D. V. Prokhorov, V. D. Stepanov, Weighted estimates for a class of sublinear operators, Dokl. Akad. Nauk 453 (2013), no. 5, 486-488, (Russian), English transl., Dokl. Math. 88 (2013), no. 3, 721-723, MR3203323.

[25] D. V. Prokhorov, V. D. Stepanov, Estimates for a class of sublinear integral operators, Dokl. Akad. Nauk 456 (2014), no. 6, 645-649, (Russian), English transl., Dokl. Math. 89 (2014), no. 3, 372-377, MR3287911.

[26] D. V. Prokhorov, On the boundedness of a class of sublinear integral operators, Dokl. Akad. Nauk 92 (2015), no. 2, 602-605, (Russian).

[27] E. PUstylnik, Optimal interpolation in spaces of Lorentz-Zygmund type, J. Anal. Math. 79 (1999), 113-157, DOI 10.1007/BF02788238, MR1749309 (2001a:46028). 
[28] G. Sinnamon, V. D. Stepanov, The weighted Hardy inequality: new proofs and the case $p=1$, J. London Math. Soc. (2) 54 (1996), no. 1, 89-101, DOI 10.1112/jlms/54.1.89, MR1395069 (97e:26021).

[29] G. È. Shambilova, Weighted inequalities for a class of quasilinear integral operators on the cone of monotone functions, Sibirsk. Mat. Zh. 55 (2014), no. 4, 912-936, (Russian, with Russian summary), English transl., Sib. Math. J. 55 (2014), no. 4, 745-767, MR3242605.

[30] V. D. Stepanov, G. È. Shambilova, Weight boundedness of a class of quasilinear operators on the cone of monotone functions, Dokl. Math. 90 (2014), no. 2, 569-572. 